ИЗВЕСТИЯ АКАДЕМИИ НАУК ЭСТОНСКОП ССР. ТОМ 27 ФИЗИКА * МАТЕМАТИКА. 1978, № 4

\title{
ВЛИЯНИЕ ДИФРАКЦИИ ОПОРНОИ ВОЛНЫ НА ГОЛОГРАФИЧЕСКИЙ ПРОЦЕСС
}

\author{
(Представлена П. Кардом)
}

Лазерное излучение с волновым числом $k=2 \pi / \lambda$ падает в виде плоской волны единичной амплитуды на амплитудно-фазовый фильтр $\left[{ }^{1}\right]$ с круговой симметрией и пропусканием $c(\varrho)$, заданным в круге $0 \leqslant \varrho \leqslant a=$ $=10^{5} / k=1 \mathrm{~cm}$. Фильтр представляет собой объект.

Пусть фильтр состоит из двух компонентов с разным пропусканием. Тогда амплитуда в точке картины с координатой $v$ выразится в виде суперпозиции $\left[{ }^{1}\right]$ :

$$
\begin{aligned}
u_{0}\left(v, c_{1}, c_{2}, a_{1}\right) & =\sum_{i=1}^{2}\left\{c_{i} \int_{a_{t-1}}^{a_{t}} J_{0}(v \varrho) \varrho d \varrho\right\}= \\
& =\left(c_{1}-c_{2}\right) a_{1} \frac{J_{1}\left(a_{1} v\right)}{v}+c_{2} \frac{J_{1}(v)}{v},
\end{aligned}
$$

где $J_{0}, J_{1}$ - функции Бесселя порядка 0 и $1 ; v=k \alpha(\alpha-$ угловое расстояние точки наблюдения от центра картины); $a_{1}$ - радиус круга, разделяющего компоненты фильтра $\left(a_{1}<1\right) ; c_{1}-$ пропускание фильтра в зоне $0 \leqslant \varrho \leqslant a_{1} ; c_{2}$ - то же в зоне $a_{1} \leqslant \varrho \leqslant a_{2}=a=1$.

На окружающую данный фильтр область $a \leqslant a_{K} \leqslant 0 \leqslant a_{L} \leqslant \infty$ падает такое же лазерное излучение, ослабляемое в общем случае во всех точках в одинаковой степени (учитывается соответствующим коэффициентом $\left.c_{0} \leqslant 1\right)$. Опорную волну представляет дифрагированная в этой области волна, влџяние которой на точку картины с координатой v выражается формулой

$$
\begin{aligned}
u_{t}\left(v, a_{K}, a_{L}, c_{0}\right) & =c_{0} \int_{a_{K}}^{a_{L}} J_{0}(v \varrho) \varrho d Q= \\
& =c_{0}\left[a_{L} \frac{J_{1}\left(a_{L} v\right)}{v}-a_{K} \frac{J_{1}\left(a_{K} v\right)}{v}\right] .
\end{aligned}
$$

Результирующая амплитуда в точке голограммы (эмульсии фотопластинки) с координатой $v$ выражается формулой

$$
u(v)=u_{0}\left(v, c_{i}, a_{1}\right)+u_{t}\left(v, a_{K}, a_{L}, c_{0}\right),
$$

а интенсивность - формулой

$$
I(v)=I\left(v, c_{i}, a_{1}, a_{K}, a_{L}, c_{0}\right)=u u^{*}=u_{0} \dot{u}_{0}^{*}+u_{t} u_{t}^{*}+u_{0} \dot{u_{t}}+\dot{u_{0}} u_{t} .
$$


Рис. 1.

Результаты вычислений интенсивности по этой формуле в зависимости от величины дифракции опорной волны иллюстрирует рис. 1. Предполагается, что объект не изменяет фазы, т. е. $c_{1}$ и $c_{2}$ вещественны и положительны.

Исследование дифракционного эффекта в зоне $0 \leqslant v \leqslant 10$ голограммы показывает, что с его ростом, т. е. с уменьшением $a_{L}$ от 100 до 10 (или с возрастанием $a_{K}$ ), уменьшается число максимумов

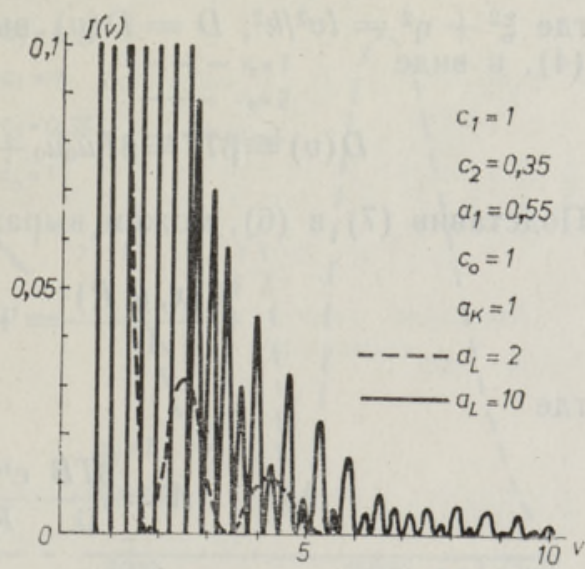
интенсивности и изменяются отношения их высот. При наибольшей дифракции $\left(a_{K}=1, a_{L}=2\right)$ число этих максимумов в рассматриваемой зоне становится примерно в пять раз меньше, чем при $a_{L}=10, a_{K}=1$ или 2. В случае наименьшего дифракционного эффекта опорной волны (если $a_{L}=100, a_{K}=1$ или 2) число наблюдаемых на голограмме максимумов (минимумов) и отношения их высот можно приближенно принять за структуру голограммы, характерную для объекта. В данном случае в каждой зоне $v_{i} \leqslant v \leqslant v_{i}+1$ (если $v_{i}=0,1,2,3, \ldots$ ) голограммы поглощающего объекта с двумя компонентами наблюдается 31 максимум интенсивности (на рис. 1 они не показаны), которые обусловлены членами интерференции $u_{0} u_{t}$ объектной и опорной волн в распределении (4). Притом значения нх максимумов быстро убывают с ростом $v_{i}$ $\left(v_{i}=0,1,2,3, \ldots\right)$ согласно приближенному закону $1: 1,3 \cdot 10^{-1}$ : $: 1,3 \cdot 10^{-3}: 3 \cdot 10^{-4}: \ldots$

Указанное число максимумов остается почти таким же при переходе от амплитудного объекта к фазовому (в котором световая фаза изменяется на $\pi$ ); становятся другими лишь расположение максимумов и отношение их высот.

В плоскости голограммы $(\xi, \eta)$ распределение пропускания фотоэмульсии дается формулой $\left(\left[{ }^{2}\right]\right.$, с. 118$)$

$$
D(\xi, \eta)=\beta T I=\beta T u u^{*},
$$

где коэффициент $\beta$ определяется свойствами фотоэмульсии, $T-$ время экспозиции.

Для восстановления оптического изображения голограмма с пропусканием (5) освещается лазерным излучением (с волновым числом $k$ ). Плоская волна, дифрагированная на голограмме, дает на расстоянии $R$ от голограммы в плоскости $(x, y)$, параллельной плоскости голограммы, распределения амплитуды $\Psi(x, y, R)$. Применяя для нахождения $\Psi(x, y, R)$ теорию дифракции Кирхгофа $\left(\left[{ }^{3}\right]\right.$, с. $\left.345 ;\left[{ }^{2}\right]\right)$, в случае френелевской дифракции получим формулу

$$
\begin{gathered}
\Psi(x, y, R)=\frac{B}{i \lambda} \frac{\mathrm{e}^{i k R}}{R} \mathrm{e}^{i \frac{k}{2 R}\left(x^{2}+y^{2}\right)} \times \\
\times \iint D(\xi, \eta) \mathrm{e}^{i \frac{k}{2 R}\left(\xi^{2}+\eta^{2}\right)} \mathrm{e}^{-i \frac{k}{R}(x \xi+y \eta)} d \xi d \eta,
\end{gathered}
$$


где $\xi^{2}+\eta^{2}=l v^{2} / k^{2} ; D=D(v)$ выражается, согласно формулам (5) и (4), в виде

$$
D(v)=\beta T I=\beta T u_{0} u_{0}^{*}+\beta T u_{t} u_{t}^{*}+\beta T u_{0} u_{t}^{*}+\beta T u_{0}^{*} u_{t}
$$

Подставив (7) в (6), можем выразить Ч как сумму четырех членов:

$$
\frac{\Psi(x, y, R)}{A}=\Psi_{1}+\Psi_{2}+\Psi_{3}+\Psi_{4},
$$

где

$$
A=\frac{\beta T B}{i \lambda} \cdot \frac{\mathrm{e}^{i k R}}{R} \mathrm{e}^{i \frac{k}{2 R}\left(x^{2}+y^{2}\right)} .
$$

Для восстановления оптического изображения существенное значение имеют члены $\Psi_{3}$ и $\Psi_{4}$ выражения (8), которые, отражая взаимосвязь объектной и опорной волн, представляют действительное и мнимое изображения. В случае вещественных $c_{i}$ верно $\Psi_{3}=\Psi_{4}$.

Поскольку $D(v)$ обладает круговой симметрией, целесообразно перейти к полярным координатам $v, \varphi$. Тогда, согласно (6), в направлении, перпендикулярном плоскости голограммы (при $x=0, y=0)$, $\Psi$ принимает вид (после интегрирования по $\varphi$ )

$$
\frac{\Psi(0,0, R)}{2 \pi A}=\int_{0}^{v_{0}} I(v) \mathrm{e}^{i \frac{l v^{2}}{2 k R}} v d v
$$

где $v_{0}$ определяет величину освещенной области голограммы.

Подставляя в третий (или четвертый) член уравнения (7) формулы (1) и (2), находим

$$
\Psi_{3}(0,0, R)=\Psi_{3}(R)+i \Psi_{3 i m},
$$

где $\left[{ }^{4}\right]$

$$
\begin{gathered}
\Psi_{3}(R)=\Psi_{3}\left(R, c_{1}, c_{2}, a_{1}, c_{0}, a_{K}, a_{L}\right)= \\
=c_{0}\left(c_{1}-c_{2}\right) a_{1} a_{L} \int_{0}^{v_{0}} \frac{J_{1}\left(a_{1} v\right)}{v} J_{1}\left(a_{L} v\right) \cos \left(\frac{l v^{2}}{2 k R}\right) d v- \\
-c_{0}\left(c_{1}-c_{2}\right) a_{1} a_{K} \int_{0}^{v_{0}} \frac{J_{1}\left(a_{1} v\right)}{v} J_{1}\left(a_{K} v\right) \cos \left(\frac{l v^{2}}{2 k R}\right) d v+ \\
+c_{0} c_{2} a_{L} \int_{0}^{v_{0}} \frac{J_{1}(v)}{v} J_{1}\left(a_{L} v\right) \cos \left(\frac{l v^{2}}{2 k R}\right) d v- \\
-c_{0} c_{2} a_{K} \int_{0}^{v_{0}} \frac{J_{1}(v)}{v} J_{1}\left(a_{K} v\right) \cos \left(\frac{l v^{2}}{2 k R}\right) d v .
\end{gathered}
$$

Входящие в формулу для $\Psi_{3}(R)$ интегралы 


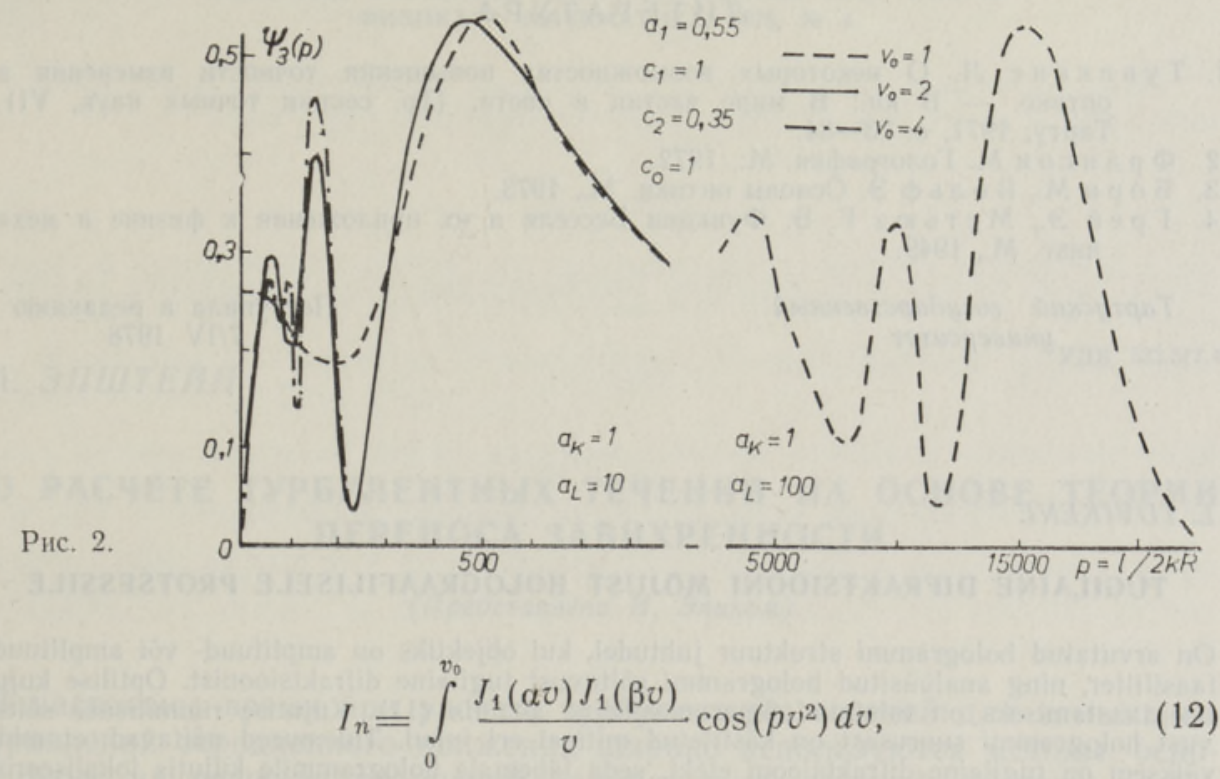

где $p=l / 2 k R$, при $v_{0}=1,2,4$ вычислялись с помощью ӘВМ. В случае $v_{0}=\infty$ интеграл этого типа был представлен в виде разложения в гипергеометрические ряды. Оказалось, что $\Psi_{3}\left(R, v_{0}=\infty\right)$ мало отличается от $\Psi_{3}\left(R, v_{0}=4\right)$.

На рис. 2 показаны для некоторых частных случаев амплитудные распределения $\Psi_{3}(p)$, вычисленные вышеизложенным способом.

В результате расчетов выяснилось следующее. Чем меньше дифракция опорной волны (т. е. чем больше $a_{L}$ и меньше $a_{K}$ ), тем ближе к голограмме локализуется восстановленное оптическое изображение (отличное от нуля амплитудное распределение) и тем дальше оно простирается вдоль нормали голограммы (с несколькими максимумами). При этом расширяется главный максимум, который практически определяет глубину и объемность фокусировки изображения.

В случае наибольшего дифракционного эффекта опорной волны $\left(a_{K}=1, a_{L}=2\right)$ восстановленное изображение локализуется очень далеко от голограммы $(0 \leqslant p<500)$. Если при восстановлении изображения освещается область $0 \leqslant v \leqslant v_{0}=2$, главный максимум находится при $p=50$; если же $v_{0}=1$, он смещается в точку $p=0$ $(R=\infty)$.

Существенную роль при восстановлении оптического изображения играют величина освещенной области голограммы и ее расположение. Чем больше эта область, тем яснее проступают побочные максимумы вдоль нормали голограммы. Известно, что в случае малого дифракционного эффекта опорной волны значения характерных для объекта максимумов ннтенсивности на голограмме особенно велики в промежутке $0 \leqslant v \leqslant 1$. Эта область голограммы и определяет действительную структуру восстановленного изображения; существенное дополнение к изображению дает также область $1 \leqslant v_{0} \leqslant 2$; остальная часть голограммы $2 \leqslant v_{0}<\infty$ уточняет лишь тонкую структуру изображения.

При переходе от амплитудного объекта к фазовому изменения в структуре восстановленного. изображения незначительны. 


\section{ЛИТЕРА Т У Р А}

1. Тувикене Л. О некоторых возможностях повышения точности измерения в оптике. - В кн.: В мире частиц и света. (Тр. секции точных наук, VI). Тарту, 1971 , с. $73-84$.

2. Фр ан сон М. Голография. М., 1972 .

3. Борн М., В ольф Э. Основы оптики. М., 1973.

4. Г рей Э., М е тьюз Г. Б. Функции Бесселя и их приложения к физике и механике. М., 1949.

\section{Тартуский государственный университет}

\section{Поступила в редакцию $7 /$ IV 1978}

\section{TUVIKENE}

\section{TUGILAINE DIFRAKTSIOONI MOJUST HOLOGRAAFILISELE PROTSESSILE}

On arvutatud hologrammi struktuur juhtudel, kui objektiks on amplituud- või amplituudfaasifilter, ning analüüsitud hologrammi sōltuvust tugilaine difraktsioonist. Optilise kujutise taastamiseks on tuletatud 6-parameetriline avaldis (11). Kujutise ruumilisuse sōltuvust hologrammi suurusest on käsitletud mitmel eri juhul. Tulemused näitavad, et mida väiksem on tugilaine difraktsiooni efekt, seda lähemale hologrammile kujutis lokaliseerub ja seda laiemale alale piki hologrammi normaali ta laotub.

\section{TUVIKENE}

\section{EFFECT OF REFERENCE WAVE DIFFRACTION ON THE HOLOGRAPHIC PROCESS}

The structure of the hologram is calculated in case amplitude and the phase-amplitude filter serve as research objects. The dependence of the hologram on reference wave diffraction is analysed. For the reconstruction of the optical image an expression (11) of 6 parameters has been derived. The dependence of the spatial image on the magnitude of the hologram has been studied in several special cases. The results demonstrate that the smaller the effect of reference wave diffraction the closer the image is located to the hologram and the wider is the area which it occupies along the normal of the hologram. 